ISSN 2763-6860

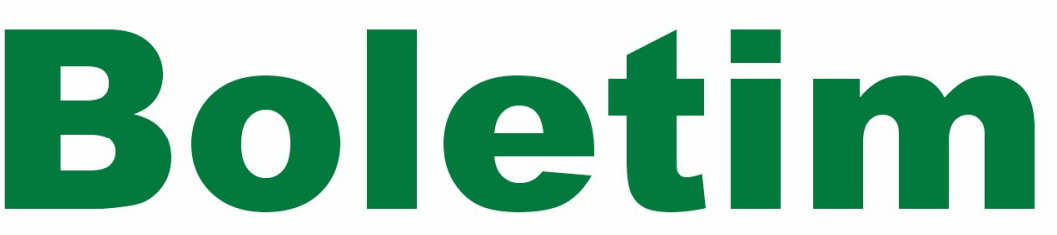

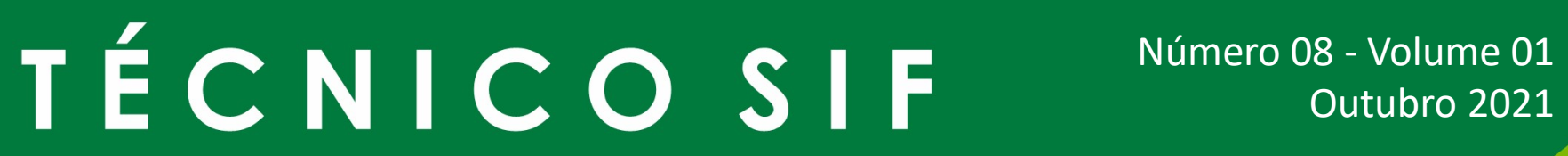

INSERÇÃO TECNOLÓGICA DOS CLONES HIIBRIDOS DE CORYMBIA SPP.NA PRODUÇÃO DE POLPA CELULÓSICA - PARTE 1: POLPAÇÃO KRAFT MODIFICADA LO-SOLIDS

Marcelo Moreira da Costa et. al. 


\title{
INSERÇÃO TECNOLÓGICA DOS CLONES HÍBRIDOS DE CORYMBIA SPP. NA PRODUÇÃO DE POLPA CELULÓSICA - PARTE 1: POLPAÇÃO KRAFT MODIFICADA LO-SOLIDS
}

\author{
Marcelo Moreira da Costa ${ }^{2 *}$, Larissa Soares Silva ${ }^{3}$, Weslley Henrique Martins da Silva ${ }^{3}$, Rodrigo Fraga de Al- \\ meida $^{4}$, Marcela Ribeiro Coura ${ }^{4}$, Gleison Augusto dos Santos ${ }^{2}$, Lilian Alves Carvalho Reis ${ }^{5}$, Claudilene Apare- \\ cida Alves Pena ${ }^{5}$ e Rayanne Oliveira Teixeira ${ }^{5}$

\footnotetext{
${ }^{2}$ Universidade Federal de Viçosa, Departamento de Engenharia Florestal, Viçosa, MG - Brasil. E-mail: <mmcd@ufv.br>e < gleison@ufv. br>.

${ }^{3}$ Universidade Federal de Viçosa, Graduando em Engenharia Florestal, Viçosa, MG - Brasil. E-mail: < larissa.s.silva@ufv.br $>$ e $<$ weslley. silva@ufv.br>.

${ }^{4}$ Universidade Federal de Viçosa, Laboratório de Celulose e Papel, Viçosa, MG - Brasil. E-mail: <rodrigo.almeida@ufv.br $>$ e $<$ coura. marcela@hotmail.com>.

${ }^{5}$ Aperam BioEnergia, Gerência Técnica Florestal Capelinha, MG - Brasil. E-mail: <lilian.reis@aperam.com>,<claudilene.pena@aperam. com>e $<$ rayanne.teixeira@aperam.com>.

*Corresponding author.
}

RESUMO - O crescimento e a consolidação das indústrias produtoras de polpa celulósica no Brasil, sofreram mudanças com o passar dos anos. Atualmente, a produtividade máxima que antes era tomada como principal objetivo, tem como aliada critérios que visam adequar a madeira para fabricação do produto desejado. Portanto, materiais genéticos superiores de maior produtividade florestal e menor consumo especifico de madeira com baixo consumo de reagentes e alto rendimento com qualidade, constituem a demanda do mercado atual. Neste contexto, a busca por biomassas como fonte fibrosa alternativa semelhante à fibra de Eucalyptus spp. tem grande valor com aplicação em escala comercial, que toca na interface floresta-indústria na produção de polpa kraft branqueada de mercado. O presente trabalho tem como objetivo apresentar os híbridos de Corymbia spp., clones desenvolvidos pela Aperam BioEnergia, como potencial substituto da madeira de Eucalyptus spp. fonte fibrosa na indústria produtora de polpa celulósica.. Por meio da realização de cozimentos kraft modificados Lo-Solids, análises da densidade básica e da morfologia das fibras, foi possível comparar as biomassas de Eucalyptus spp. vs. Corymbia spp., mediante seus respectivos IMAcell e consumo

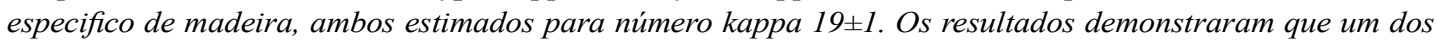
clones do híbrido de Corymbia citriodora x Corymbia torelliana (ID 4), apresentou maior densidade básica, maior rendimento depurado, melhor IMAcel ${ }_{\text {\#k19 }}$ e menor consumo especifico de madeira, quando comparada às demais amostras, atingindo valores de 0,608 t. $\mathrm{m}^{-3}, 54,1 \%, 24,6 \mathrm{tsa} \cdot \mathrm{ha}^{-1}$.ano $\mathrm{o}^{-1}$ e 2,74 $\mathrm{m}^{3} . \mathrm{ss}^{-1}$, respectivamente. Consequentemente, o alinhamento dos melhores resultados desse clone (ID 4), levou ao maior valor no ranqueamento de 8,98, estabelecido pela relação entre IMAcell/CE ${ }_{\text {Mad }}$ Mostrando a produtividade florestal superior, aliada ao menor custo industrial que as demais matérias-primas, justificou seu uso com vantagens frente à madeira de Eucalyptus spp, também avaliadas neste trabablho.

Palavras-Chave: Hibridos de Corymbia; Polpação Kraft Lo-Solids; Produtividade florestal; Consumo especifico de madeira e Ranqueamento dos clones comerciais. 


\section{INTRODUÇÃO}

Atualmente, a indústria brasileira produtora de celulose branqueada de mercado, maior exportadora global, exibe uma tendência muito forte do aumento da capacidade de produção. Em outras palavras, existe uma demanda significativa de madeira como fonte fibrosa no Brasil, com custos operacionais da madeira iguais ou menores, bem como qualidade igual ou superior à madeira de eucalipto. As madeiras de várias espécies de Eucalyptus ssp., e seus clones, apresentam alta qualidade para produção de diferentes tipos de papéis, e sua produtividade florestal impressiona, quando comparada à outras espécies florestais.

O setor brasileiro produtor de celulose de mercado tem perspectiva de incremento da escala de produção de forma significativa. Este fato levou nos últimos anos o Brasil a ser o maior exportador mundial de polpa kraft branqueada de mercado, vinda da madeira de Eucalyptus spp. (BEKP). A polpa de fibra curta brasileira tem sido usada para diferentes tipos de papéis ( $\mathrm{P} \& W$; Tissue e Embalagens), sendo exportados cerca de $15 \mathrm{Mi}$ tsa/ano. Segundo o Relatório do IBA 2019 o saldo da balança comercial do setor de árvores plantadas equivale à 12,5 Mi US\$/ ano, onde $75,2 \%$ advém da indústria produtora de polpa celulósica e papel (IBÁ, 2019).

Portanto, a possível baixa disponibilidade de madeira, fonte fibrosa de boa qualidade, é previsto nos próximos anos. Diante deste cenário, se faz necessário estudar alternativas de biomassas semelhantes a fibra de Eucalyptus spp. Industrialmente, no setor produtor de polpa celulósica e papel, a utilização da madeira dos clones híbridos de Corymbia spp. foi muito pouco explorada até o momento. As espécies de Corymbia spp. vêm despontando neste contexto, como fonte de matéria-prima alternativa, porém, o setor industrial ainda tem fortes restrições do uso deste material. O desenvolvimento tecnológico é essencial, e deve estabelecer os principais parâmetros de processo, viabilizando sua utilização. Com essa finalidade, importante levantar entre outros parâmetros o IMAcel $\left(\right.$ tsa.ha $^{-1} \cdot$ ano $\left.^{-1}\right)$ no qual diz respeito à produtividade florestal, permitindo ranquear os clones comerciais, com base na produção de polpa celulósica por unidade de área plantada ao longo do período de plantio. Em adição, o consumo especifico de madeira $\left(\mathrm{CE}, \mathrm{m}_{\mathrm{Mad}}^{3}{ }^{\prime}\right.$ tsa) ajuda a prever os custos operacionais na área fabril.
Em virtude ao processamento dos híbridos de Corymbia spp. para produção de polpa celulósica, ressalta-se a aplicação do processo kraft modificado Lo-Solids, a fim de aumentar a seletividade do processo de polpação voltados para madeiras de alta densidade. Esse processo apresenta impregnação apropriada dos cavacos com divisão da carga alcalina, evitando parte da degradação dos carboidratos, via dissolução de hemiceluloses, reações de despolimerização terminal e por meio da hidrólise alcalina de ligações glicosídicas, levando a perda de viscosidade e rendimento (Gomide, 1979 e Santos, 2008). Desta forma, a polpação kraft modificada Lo-Solids conduz uma redução dos picos de concentração de álcali, redução da temperatura de reação, minimiza a concentração de lignina residual e maximiza a sulfidez na fase inicial. Para tanto, o licor negro é extraído várias vezes durante o processo de polpação, sendo cada extração seguida pela adição de licor branco, mantendo as concentrações de licores desejadas.

Esse trabalho visa desenvolver alternativas tecnológicas voltadas a mitigar os principais gargalos na utilização da madeira de clones dos híbridos de Corymbia spp., com elevada densidade básica. Por fim, realizar ranqueamento dos clones comerciais de Eucalyptus spp., frente aos clones dos híbridos de Corymbia spp., em alusão à produtividade florestal via IMAcel e adicionalmente ao consumo específico de madeira, utilizando o protocolo industrial de polpação kraft modificada Lo-Solids.

\section{MATERIAIS E MÉTODOS}

Para a execução deste estudo, foram analisadas 16 amostras de cavacos provenientes das unidades industriais da empresa Aperam Bioenergia, no Vale do Jequitinhonha em Minas Gerais.. A descrição das amostras com 6,5 anos, e suas respectivas identificações, são fornecidas na Tabela 1 .

\subsection{Polpação Kraft modificada Lo-solids}

O procedimento de polpação foi realizado no digestor MK, modelo 610-2, que possui 2 reatores com capacidade de 7 litros com controle eletrônico. Foi empregado uma massa de 400 gramas secas de cavacos no digestor MK, aquecido eletricamente e dotado de controles eletrônicos ligados ao computador. Os dados de temperatura foram monitorados a cada minuto durante o procedimento de polpação, possibilitando estabelecer

Boletim Técnico SIF 2021:01 
Tabela 1 -Identificação dos materiais genéticos utilizados na avaliação em escala de laboratório com 6,5 anos.

\begin{tabular}{ll}
\hline ID & Material genético - 6,5 anos \\
\hline 1 & Corymbia citriodora $x$ Corymbia torelliana \\
2 & Corymbia citriodora $x$ Corymbia torelliana \\
3 & Corymbia citriodora $x$ Corymbia torelliana \\
4 & Corymbia citriodora $x$ Corymbia torelliana \\
5 & Eucalyptus cloeziana \\
6 & Eucalyptus urophylla $x$ Eucalyptus spp. \\
7 & Eucalyptus urophylla $x$ Eucalyptus spp. \\
8 & Eucalyptus urophylla $x$ Eucalyptus spp. \\
9 & Eucalyptus grandis $x$ Eucalyptus urophylla \\
10 & Eucalyptus urophylla $x$ (Eucalyptus \\
& camaldulensis $x$ Eucalyptus grandis) \\
11 & (Eucalyptus camaldulensis $x$ Eucalyptus \\
& grandis) $x$ Eucalyptus urophylla \\
12 & (Eucalyptus camaldulensis $x$ Eucalyptus \\
& grandis) $x$ Eucalyptus urophylla \\
13 & (Eucalyptus camaldulensis $x$ Eucalyptus \\
& grandis) $x$ Eucalyptus urophylla \\
14 & (Eucalyptus camaldulensis $x$ Eucalyptus \\
15 & grandis) $x$ Eucalyptus urophylla \\
& (Eucalyptus camaldulensis $x$ Eucalyptus \\
& grandis) $x$ Eucalyptus urophylla \\
& Eucalyptus urophylla $x$ Eucalyptus pellita \\
\hline
\end{tabular}

o perfil de temperatura e de fator $\mathrm{H}$ ao final do processo. As cargas de álcali efetivo (AE, \%) foram aplicadas como $\mathrm{NaOH}$, base massa seca de madeira, e selecionadas após realização de testes exploratórios, visando o número kappa $19 \pm 1$. A polpação kraft modificada Lo-solids foi realizada nas seguintes etapas a saber: (1) Prévaporização dos cavacos realizada com vapor de baixa pressão (3,5 bar) por 15 minutos, onde a temperatura da superfície do cavaco foi aumentada para cerca de $105^{\circ} \mathrm{C}$; (2) Impregnação dos cavacos foi obtida pela adição de $55 \%$ da carga total de álcali efetivo (AE), seguido pelo incremento da temperatura de $105^{\circ} \mathrm{C}$ para $135^{\circ} \mathrm{C}\left(15^{\prime}\right.$ de rampa), mantidos por 90'; (3) Cozimento foi realizado pela drenagem do volume de licor de impregnação e posteriormente injeção por deslocamento do licor branco, perfazendo $45 \%$ da carga total de $\mathrm{AE}$, onde a temperatura foi elevada de $135^{\circ} \mathrm{C}$ para $165^{\circ} \mathrm{C}$, em $10^{\prime}$ de rampa. A temperatura foi mantida a $165^{\circ} \mathrm{C}$ por $90^{\prime}$ como tempo de reação com relação licor/madeira de 4/1; (4) A Lavagem foi realizada ao término deste período, e o licor preto foi extraído e por deslocamento. O processo de lavagem foi realizado em duas etapas, a primeira consiste em uma lavagem alcalina à $3 \%$ de $\mathrm{AE}$, na qual a temperatura foi reduzida para $90^{\circ}$, por $60^{\circ}$ e na sequencia extraído. Posteriormente, a lavagem foi realizada com água quente a $90^{\circ} \mathrm{C}$ por 15 , momento em que a temperatura foi reduzida para $40^{\circ} \mathrm{C}, \operatorname{com} 9 \mathrm{~m}^{3} /$ tsa.

\subsection{Procedimentos Analíticos}

Para a caracterização dos cavacos, licor preto e polpa celulósica marrom produzida, foram realizadas a seguintes análises e suas respectivas normas: Densidade básica (DB) - ABNT (NBR 11941); número kappa (\#K) - Tappi T $236 \mathrm{~cm}-85$; pH do licor preto - Tappi T211 om-93; álcali efetivo residual do licor preto - SCAN N2:88; rendimento depurado e rejeitos da polpação Lo-Solids - Gravimetria (Norma interna LCP); e Morfologia da polpa celulósica Protocolo completo da Valmet Fiber Image Analyzer (Valmet FS5). O consumo de álcali efetivo (AE\#k19) e rendimento depurado $\left(\mathrm{RD}_{\text {\#k19 }}\right)$ para número kappa $19 \pm 1$ foram estimados, a partir de três testes de polpação kraft modificada Lo-Solids, com variação da carga de AE aplicada, mantendo-se as demais condições constantes. O IMAcel foi obtido através do produto entre os valores do volume do incremento médio anual (IMA), rendimento depurado em polpa celulósica e densidade básica das amostras de cavaco. $\mathrm{O}$ ranqueamento das amostras foi estabelecido de acordo com relação entre os valores de IMAcel e do consumo específico de madeira, ambos usando polpação Lo-Solidos estimado para número kappa $19 \pm 1$.

\section{RESULTADOS E DISCUSSÃO}

Mediante as análises de densidade básica das amostras de madeiras apresentadas na Tabela 2, podemos evidenciar que a madeira de Corymbia citriodora x Corymbia torelliana (ID 1 e 4) apresentam maior valor de densidade básica, equivalente à 0,608 t.m ${ }^{-3}$. Além disso, destaca-se a elevada densidade do Eucalyptus cloeziana. O menor valor de densidade básica foi observado para amostra (Eucalyptus camaldulensis x Eucalyptus grandis) x Eucalyptus urophylla (ID 14).

Os resultados obtidos na polpação kraft modificada, evidenciaram que os clones de Corymbia citriodora x Corymbia torelliana (ID 3 e ID 4), apresentaram o menor consumo de $\mathrm{AE}_{\# \mathrm{k} 19}$ e o maior valor de $\mathrm{RD}_{\text {抽19 }}$, entre as amostras avaliadas (Tabela 2). Por outro lado, a amostra de Eucalyptus cloeziana (ID 5) apresentou o pior desempenho, posto que necessitou da maior carga de álcali efetivo e consequentemente resultou em menor rendimento de polpa celulósica em relação as demais amostras analisadas neste trabalho. 
Na Tabela 2 evidencia-se que o maior valor de IMAcel foi observado para a amostra de Corymbia citriodora x Corymbia torelliana (ID 4), seguida pela amostra Eucalyptus urophylla x Eucalyptus spp (ID 8 ), respectivamente com valores de 24,6 e 17,5 . O menor valor de produtividade florestal, i.e., IMAcell, encontrado para as amostras avaliadas foi observado também para uma amostra de Corymbia citriodora x Corymbia torelliana (ID 1) com valor de 10,3 adt ha $^{-1}$. ano ${ }^{-1}$. Este fato, confirma a importância da prévia caracterização das diferentes matérias-primas fonte fibrosa para indústria de polpa kraft branqueada de mercado. Em adição, o menor consumo especifico de madeira foi estabelecido para a amostra de Corymbia citriodora x Corymbia torelliana (ID 4). Consequentemente, esse mesmo clone de híbrido de Corymbia spp. foi aquele que apresentou maior valor no ranqueamento pois apresento maior IMAcel e menor $\mathrm{CE}_{\mathrm{Mad}}$. Portanto, esse material mostrou excelente potencial tanto na produtividade florestal quanto no custo operacional com base no consumo especifico de madeira.

De acordo com os resultados da Tabela 2, evidenciou-se o impacto positivo e significativo dos maiores valores de densidade básica da madeira de Corymbia spp. nos valores de IMAcel e no CEMad. Em particular, o clone de Corymbia citriodora $\mathrm{x}$
Corymbia torelliana (ID 4), mostrou excelente desempenho não somente na produtividade florestal, bem como nos valores referentes a polpação kraft,

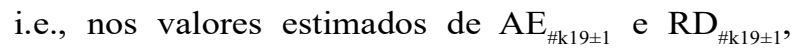
mostrando ser uma potencial escolha para plantio comercial, voltado ao alto desempenho na interface floresta-indústria.

Adicionalmente, a Tabela 3 mostra os valores obtidos da morfologia das fibras da polpa marrom de todos os clones avaliados neste trabalho. Percebe-se que a amostra de Corymbia citriodora x Corymbia torelliana (ID 1 e ID 4) apresentou, ligeiramente maior comprimento médio de fibras. Este fato pode ser melhor explorado para polpas voltadas para papéis de maiores resistências mecânicas, principalmente buscando maiores índices de tração. Certamente, essas propriedades físico-mecânicas serão melhor exploradas, na parte II desta pesquisa, onde as polpas marrons vindas do processo Lo-Solids serão branqueadas e posteriormente refinadas em moinho laboratorial PFI para avaliação dos 16 clones da Aperam.

\section{CONCLUSÃO}

O ritmo de novos investimentos voltados a produção de polpa kraft branqueada de mercado no

Tabela 2 - Resultados de demanda de álcali efetivo (AE\%), rendimento depurado da polpa, teor de sólidos secos, IMAcel e consumo especifico de madeira estimado para $\# K=19 \pm 1$

\begin{tabular}{|c|c|c|c|c|c|c|c|}
\hline$\overline{\mathrm{ID}}$ & $\begin{array}{c}\mathrm{DB} \\
\left(\mathrm{t} \mathrm{m}^{-3}\right) \\
\end{array}$ & $\begin{array}{c}\mathrm{AE} \\
(\%)^{1}\end{array}$ & $\begin{array}{l}\mathrm{RD} \\
(\%)^{1}\end{array}$ & $\begin{array}{c}\text { IMA } \\
\left(\mathrm{m}^{3} \cdot \text { ha }^{-1} \cdot \text { ano }^{-1}\right)^{2}\end{array}$ & $\begin{array}{c}\text { IMAcell } \\
\left(\text { adt ha }{ }^{-1} \cdot \text { ano }^{-1}\right)^{3}\end{array}$ & $\begin{array}{c}\mathrm{CE}_{\mathrm{Mad}} \\
\left(\mathrm{m}^{3}{ }_{\mathrm{Mad}} \cdot \mathrm{adt}^{-1}\right)^{3}\end{array}$ & $\begin{array}{c}\text { Ranqueamento } \\
\text { dos clones }{ }^{4}\end{array}$ \\
\hline$\overline{1}$ & 0,608 & 16,1 & 52,5 & 29,0 & 10,3 & 2,82 & 3,65 \\
\hline 2 & 0,565 & 15,9 & 51,6 & 34,8 & 11,3 & 3,09 & 3,66 \\
\hline 3 & 0,507 & 15,4 & 53,5 & 51,8 & 15,6 & 3,32 & 4,70 \\
\hline 4 & 0,608 & 16,0 & 54,1 & 67,4 & 24,6 & 2,74 & 8,98 \\
\hline 5 & 0,582 & 18,9 & 49,8 & 45,9 & 14,8 & 3,10 & 4,77 \\
\hline 6 & 0,518 & 16,0 & 52,7 & 37,2 & 11,3 & 3,30 & 3,42 \\
\hline 7 & 0,472 & 15,8 & 53,6 & 46,0 & 12,9 & 3,56 & 3,62 \\
\hline 8 & 0,491 & 15,8 & 53,8 & 59,6 & 17,5 & 3,41 & 5,13 \\
\hline 9 & 0,470 & 15,6 & 53,2 & 42,2 & 11,7 & 3,60 & 3,25 \\
\hline 10 & 0,511 & 16,6 & 51,1 & 39,5 & 11,5 & 3,45 & 3,33 \\
\hline 11 & 0,431 & 16,8 & 51,1 & 48,2 & 11,8 & 4,09 & 2,89 \\
\hline 12 & 0,470 & 16,9 & 52,5 & 46,7 & 12,8 & 3,65 & 3,51 \\
\hline 13 & 0,488 & 17,3 & 50,9 & 53,6 & 14,8 & 3,62 & 4,09 \\
\hline 14 & 0,431 & 16,6 & 51,9 & 47,6 & 11,8 & 4,02 & 2,94 \\
\hline 15 & 0,519 & 17,6 & 50,8 & 40,0 & 11,7 & 3,41 & 3,43 \\
\hline 16 & 0,471 & 17,6 & 51,1 & 55,1 & 14,7 & 3,74 & 3,93 \\
\hline
\end{tabular}

Boletim Técnico SIF 2021:01 
Tabela 3 - Resultados da morfologia de fibra das polpas marrom com \#K19 \pm 1 .

\begin{tabular}{|c|c|c|c|c|c|c|c|c|c|}
\hline$\overline{\mathrm{ID}}$ & $\begin{array}{l}\text { Comprimento } \\
(\mathrm{mm})\end{array}$ & $\begin{array}{l}\text { Largura } \\
(\mu \mathrm{m})\end{array}$ & $\begin{array}{l}\mathrm{CWT}^{2} \\
(\mu \mathrm{m})\end{array}$ & $\begin{array}{l}\text { Corseness } \\
\mathrm{mg} / 100 \mathrm{~m}\end{array}$ & $\begin{array}{l}\text { População } \\
\text { fibrosa \#/mg }\end{array}$ & $\begin{array}{c}\text { Finos A } \\
(\%)^{1}\end{array}$ & $\begin{array}{c}\text { Finos B } \\
(\%)^{1}\end{array}$ & $\begin{array}{l}\text { Curl } \\
(\%)\end{array}$ & $\begin{array}{l}\text { Kink } \\
(1 / \mathrm{m})\end{array}$ \\
\hline 1 & 1,03 & 18,2 & 2,04 & 5,5 & 17970 & 18,9 & 0,07 & 7,1 & 2.296 \\
\hline 2 & 0,92 & 18,2 & 2,49 & 6,5 & 15981 & 20,8 & 0,07 & 6,8 & 2.394 \\
\hline 3 & 0,88 & 17,8 & 2,15 & 5,6 & 20075 & 16,6 & 0,09 & 6,3 & 2.447 \\
\hline 4 & 0,95 & 18,1 & 1,92 & 5,2 & 19992 & 17,7 & 0,09 & 6,6 & 2.252 \\
\hline 5 & 0,88 & 20,0 & 2,49 & 7,3 & 15004 & 16,6 & 0,12 & 5,5 & 2.246 \\
\hline 6 & 0,92 & 19,0 & 1,49 & 4,3 & 26051 & 17,8 & 0,14 & 6,3 & 2.313 \\
\hline 7 & 0,91 & 18,8 & 2,00 & 5,6 & 20268 & 15,7 & 0,28 & 8,5 & 2.834 \\
\hline 8 & 0,90 & 19,3 & 2,18 & 6,2 & 17732 & 16,5 & 0,19 & 7,5 & 2.725 \\
\hline 9 & 0,87 & 19,1 & 2,02 & 5,8 & 20003 & 14,4 & 0,30 & 6,4 & 2.436 \\
\hline 10 & 0,84 & 18,9 & 2,26 & 6,3 & 18281 & 18,1 & 0,21 & 6,7 & 2.673 \\
\hline 11 & 0,81 & 18,4 & 1,84 & 5,1 & 24391 & 14,2 & 0,24 & 6,4 & 2.735 \\
\hline 12 & 0,83 & 18,3 & 1,93 & 5,3 & 23192 & 14,0 & 0,15 & 8,8 & 3.359 \\
\hline 13 & 0,80 & 18,1 & 1,82 & 4,9 & 25809 & 13,4 & 0,20 & 6,1 & 2.692 \\
\hline 14 & 0,81 & 18,7 & 1,73 & 4,9 & 25342 & 15,6 & 0,31 & 7,6 & 3.090 \\
\hline 15 & 0,84 & 17,2 & 1,79 & 4,6 & 26791 & 14,6 & 0,20 & 7,8 & 2.811 \\
\hline 16 & 0,86 & 18,3 & 1,70 & 4,7 & 25173 & 13,4 & 0,28 & 7,1 & 2.778 \\
\hline
\end{tabular}

${ }^{2} \mathrm{CWT}$ : cell wall thickness

Brasil tem forte tendência de crescimento, o que pode levar a baixa disponibilidade de madeira, fonte fibrosa de boa qualidade. Diante deste cenário, madeira dos clones híbridos de Corymbia spp. vêm despontando, como natural substituto e fonte de matéria-prima alternativa, apesar dos gargalos tecnológicos. O desenvolvimento dos processos é essencial, com foco na viabilidade da utilização desta biomassa fibrosa. Os principais resultados da avaliação dos clones desenvolvidos pela Aperam BioEnergia, demonstraram os seguintes pontos a saber:

1) O híbrido de Corymbia citriodora x Corymbia torelliana, especificamente ID 4, apresentou maior valor no ranqueamento, provando ser uma excelente alternativa de alta performance na interface florestaindústria.

2) As madeiras dos híbridos de Corymbia spp. apresentam uma vantagem clara nos custos de logística e no consumo especifico da madeira $\left(\mathrm{CE}_{\mathrm{Mad}} \mathrm{m}^{3} / \mathrm{tsa}\right)$, justamente por apresentar maior densidade básica da madeira. Apresenta, entre outras vantagens na área florestal, menor custo da madeira posto fabrica (US\$/ $\mathrm{m}^{3}$ ) e na área industrial, em menor custo por tonelada de polpa celulósica produzida (US\$/tsa).

3) Em adição, é bom notar que o ranqueamento prévio dos clones comerciais tem grande aplicação com a finalidade de separar madeiras de diferentes comportamentos em escala industrial. Idealmente, deve ser realizada seguindo o protocolo de polpação kraft industrial visando harmonizar as diferenças dos testes de laboratório com as condições industriais. Nesta avaliação foi estabelecido o processo Lo-solids com estimativa dos parâmetros para número kappa 19.

4) Os clones dos híbridos de Corymbia citriodora x Corymbia torelliana (ID 1 e ID 4) proporcionaram polpa marrom, com comprimento médio das fibras, ligeiramente superior as demais amostras avaliadas.

\section{REFERÊNCIAS}

ALENCAR, Gláucia Soares Barbosa de. Estudo da qualidade da madeira para produção de celulose relacionada a precocidade na seleção de um híbrido Eucalyptus grandis x Eucalyptus urophylla. 2002. Dissertação (Mestrado em Recursos Florestais) Escola Superior de Agricultura Luiz de Queiroz, Universidade de São Paulo, Piracicaba, 2002.

IBÁ. Indústria Brasileira de Árvores. Ibá: Indústria Brasileira de Árvores 2019. Brasília, DF, 2019. 100 p. Relatóriolbá 2019.

BARRICHELO, L. E. G.; BRITO, J. O. A madeira das espécies de eucalipto como matéria-prima para a indústria de celulose e papel Brasília: PNUD/FAO/ IBDF/BRA-45. PRODEPEF, 1976. 145p. (Série Divulgação, 13).

BASSA, Ana Gabriela Monnerat Carvalho. Misturas 
de madeira de Eucalyptus grandis x Eucalyptus urophylla, Eucalyptus globulus e Pinus taeda para produção de celulose Kraft através do Processo LoSolids ${ }^{\circledR}$. 2006. Dissertação (Mestrado em Recursos Florestais) - Escola Superior de Agricultura Luiz de Queiroz, Universidade de São Paulo, Piracicaba, 2007.

BRAZ, R. L., OLIVEIRA, J. S., ROSADO, A. M., VIDAURRE, G. B., PAES, J. B., TOMAZELLO FILHO, M., LOIOLA, P. L. Caracterização anatômica, física e química da madeira de clones de Eucalyptus cultivados em áreas sujeitas à ação de ventos. Ciência da Madeira. Pelotas, v. 5, p. 127-137, 2014

Breno Assis Loureiro; Paulo Fernando Trugilho; Taiana Guimarães Arriel; Rebeca Lima et al. COMPOSIÇÃO QUÍMICA ESTRUTURAL DA MADEIRA DO CRUZAMENTO ENTRE Corymbia citriodora e Corymbia torelliana. In: ANAIS CONGRESSO BRASILEIRO DE CIÊNCIA E TECNOLOGIA DA MADEIRA, 2017, . Anais eletrônicos... Campinas, Galoá, 2017.

Celso Edmundo BochettiFoelkel; Luiz Ernesto
George Barrichelo; Augusto F. Milanez. Estudo comparativo das madeiras de Eucalyptus saligna, E. paniculata, E. citriodora, E. maculata e E. tereticornis para produção de celulose sulfato. IPEF n.10, p.17-37, 1975.

DEMUNER, Wendel Pianca. Estudo da adição de xilanas na polpação kraft e seus impactos no desempenho do processo e qualidade do produto final. 2014. 76 f. Dissertação (Mestrado em Tecnologia de Celulose e Papel) - Universidade Federal de Viçosa, Viçosa. 2014.

FANTUZZI NETO, Humberto. Wood quality of Eucalyptus for kraft pulp production.2012. 119 f. Tese (Doutorado em Manejo Florestal; Meio Ambiente e Conservação da Natureza; Silvicultura; Tecnologia e Utilização de) - Universidade Federal de Viçosa, Viçosa, 2012.

GARDNER, R. A. W.; LITTLE, K. M.; ARBUTHNOT, A. Wood and fibre productivity potential of promising new eucalypt species for coastal Zululand, South Africa.AustralianForestry, v. 70, n. 1, p. 37-47, 2007 